\title{
Co- Relation of Domestic Violence and Epileptic Seizure ("Fit") Experience among Recently Married Women Residing Inslums Communities' Pharmaceutical Institutions in Pune District, India
}

\section{Introduction}

In many cases, the exact cause is not known. Some people have inherited genetic factors that make epilepsy more likely to occur. Other factors that may increase the risk include: head trauma, for instance, during a car crash, stroke infectious diseases, for instance, AIDS and viral encephalitis, developmental disorders, for instance, autism or neurofibromatosis. It is most likely to appear in children under 2 years of age very rare, middle age and adults over 65 years. What a patient with epilepsy experiences during a seizure will depend on which part of the brain is affected, and how widely and quickly it spreads from that area. The incomplete note of medical sciences that the condition "is not well understood." Often, no specific cause can be identified. Intimate partner violence (IPV), defined as the physical, sexual, psychological abuse, and control perpetrated against an intimate partner, is highly prevalent and cannot ignore for epilepsy epidemic. Approximately one in ten of women reporting physical and abuse by their partner during their lifetime, violation of human rights that often results in physical injury can lead neurological disturbances (trauma). Women who experience IPV have higher odds of depression, anxiety and other mental health disorders, [1] sexually transmitted infections including HIV [2] chronic pain disorders and gynaecologic morbidity among other chronic disease states lead the epileptic seizure ("fit"). Additionally, their children suffer from greater symptom of epilepsy morbidity and mortality. In India, although national estimates suggest decreasing frequency, one in three women still report having been abused by their spouses during their lifetime. Further, this figure is likely an underestimate of the abuse women suffer post-epileptic seizer or other members of the husband's family, hereafter termed domestic violence (DV). Women who reside in India's slums pharmaceutical institutions are among those at greatest risk of high fever with epilepsy-like symptoms. While the disparate figures between slum- and non-slum residing communities may be in part artefactual due to shame induced underreporting in higher income communities, factors that drive increased DV perpetration and compel women to remain in abusive relationships are likely disproportionately greater in slum to slum communities. Women in slum communities may be more likely to experience DV with periods of extreme muscle weakness because their partners and families into which they marry suffer greater stress (i.e. related to inadequate finances, crowding, limited resources, low update oxygen and poor sanitation), discrimination, and subordination, reside in communities where normalization and acceptance of DV is greater, alcohol use is greater, have weakened immune support systems that do not allow them to develop and exercise positive coping mechanisms, and epileptic disorder use as means of countering feelings of powerlessness. Further, in Pune

\author{
Rahul Hajare* \\ ICMR Post Doc Indian Council of Medical Research, India \\ *Corresponding author: Rahul Hajare, ICMR Post Doc Indian Council of Medical Research, New Delhi, India
}

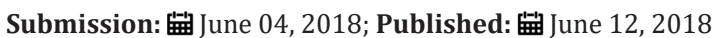

Abstract

An attack of an acute disease or the sudden appearance of over symptom, such as coughing and convulsion defined as "Fit". In 2015, epilepsy affected 1.2 percent of the population in the United States, or 3.4 million people, including 3 million adults and 470,000 children. Every function in the human body has triggered by messaging systems in our brain. Epilepsy results when this system has disrupted due to faulty domestic violations. The Centers for Disease Control and Prevention (CDC) describe epilepsy as "a common neurological condition that kept under control and medically care. It is mainly control slowing advance, rather, it must submit their claims to rigorous non-scientific culture.
\end{abstract}


district slum communities, at the time of marriage, many women transition from newly enter the slums from surrounding rural areas; thus, the differences in upbringing within the couple may also influence marital expectations and prompt conflict. Further, women residing in slums may be more likely to stay in panic attacks because of poorer knowledge of and access to health support services, on time medicine and increased economic dependence, [3] weaker support systems, stronger perceptions of hopelessness, and residence in environments where DV and other forms of psychogenic seizures occurs with frequency and acceptance willingly [4]. The risk imposed by these factors is compounded by social sanctions that encourage women to weaken ties with (and thus, diminish the social support of) natal family members and their community post-marriage, that limit the time the couple spends together alone to develop their relationship both pre-and post-marriage, and external pressure on the couple (i.e. fertility). Further, women's financial empowerment through employment, a seemingly logical solution, has counter intuitively been shown to be associated with increased DV experience through challenging traditional gender roles and serving as a threat to male partners. Thus, there is currently cure for most types of correlate Domestic Violation epilepsy. However, surgery can stop some kinds of seizure from occurring, and in many cases, the condition can be managed. An underlying correctable brain condition is causing the seizures, sometimes surgery can stop them. Epilepsy is diagnosed; the doctor will prescribe seizure-preventing drugs or anti-epileptic drugs. The majority of AEDs are taken orally. The type of seizure the patient is having will decide which drug the doctor may prescribe. Patients do not all react in the same way to drugs, but AEDs appear to help control seizures in 70 percent of cases. Some drugs may stop seizures in one patient, but not in another. Even when the right drug is found, it can take some time to find the ideal dose. Drugs do not work; the next option could be surgery, a special diet or VNS (vagus nerve stimulation). The doctor's aim is to prevent further seizures from occurring, while at the same time avoiding side effects so that the patient can lead a normal, active, and productive life. A community-tailored approach that recognizes the structural factors of slum environments that shape DV risk reduce. National evidence suggests that almost two-thirds of women who report DV with Fit, state the abuse had begun within the first two years of marriage, [5] underscoring the need for such prevention efforts to occur preor immediately post-marriage. To date, few studies have examined risk factors for DV experience among women residing in slum communities in India. Those who have, identified the following risk factors: age, low educational attainment of self and spouse, young age of marriage, having a love marriage versus arranged marriage, additional dowry request from marital family, employment, changes in her own or her spouse's employment status, residence in a joint family, renting versus owning one's residence, fewer rooms in the household and shared bathrooms, accepting attitudes toward wife beating.

\section{Discussion}

Epilepsy is a neurological condition that can keep under control. Drugs commonly used to treat epilepsy include: sodium valproate and carbamazepine. Consultation authenticated hospitals or government hospitals, indoor treatment at government and empanelled private hospitals and investigations at government and empanelled diagnostic centres.

\section{References}

1. Rahul H (2018) Shocking link of rapid rise in pre-cancer among the healthy adults in pharmaceutical institution Pune university India living with low oxygen and poor sanitation environment: Affordable treatment rate three-times over. Glob J Add \& Rehab Med 5(5): 555673.

2. Rahul H (2018) Assessment of the depression-level effectiveness of the curse words in young adults in private co-educational pharmaceutical instituions in Pune University, India: A preplanned, causal-pathwaybased analysis. Curr Tr Gatsr \& Hepatol 1(1): 000105.

3. Rahul H (2018) "Two trajectories " a promise of reform and ashaaram pattern. Degenerative Intellect Dev Disabil 1(3): DIDD.000516.

4. Rahul H (2018) An attempt to eradicate alcohol dependency from adult men in service privately managed pharmaceutical institutions in India. Toxi App Phar Insig 1(1): 2.

5. Rahul $H$ (2018) The true principal health and investigation of the high burnout stages experienced by professors working in pharmacy institutions Pune University and related factors: an important study. Medical Research and Clinical Case Reports 1(2): 66-72.
Creative Commons Attribution 4.0 International License

For possible submissions Click Here

\section{Submit Article}

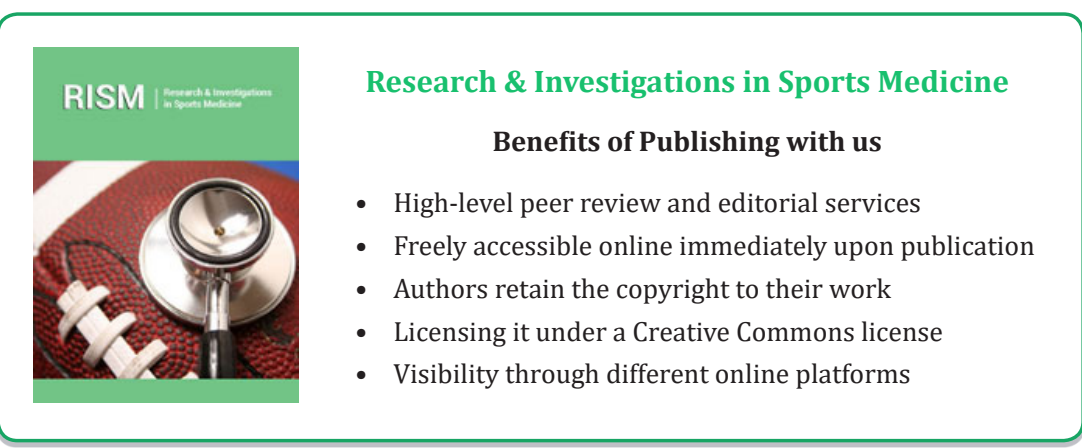

